\title{
Un siècle de peuplement en Afrique de l'Ouest
}

\author{
Fabrice Courtin ${ }^{1}$, Jean-Pierre Guengant ${ }^{2}$ \\ 1 Géographe, IRD, UMR177 (IRD/CIRAD), Centre international de recherche-développement pour l'élevage en zone subhumide \\ (CIRDES), Bobo-Dioulasso 01, Burkina Faso \\ 2 Démographe, IRD, UMR201 Développement et sociétés (IRD/Université Paris 1), 94736 Nogent-sur-Marne, France
}

\begin{abstract}
Géographe et démographe, les auteurs ont conjugué leurs regards pour présenter une narration critique de l'accroissement par facteur 10 de la population de l'Afrique de l'Ouest en 100 ans. Tout indique qu'un triplement va suivre d'ici 2050. La saturation foncière est déjà, et sera, une cause majeure de violentes crises sociales. Si l'espoir est de prolonger les situations et les pratiques actuelles, les politiques disponibles (double révolution verte, contrôle des naissances et adaptation au changement climatique) ne seront pas suffisantes. Les auteurs appellent à un examen de ces défis énormes qui s'exprimeront selon eux au travers de révolutions économique, sociale et démographique, dont les issues seront fortement dépendantes des contextes internationaux.
\end{abstract}

La Rédaction

\section{Mots-clés :}

peuplement ;

climat ;

géographie ;

démographie ;

Afrique de l'Ouest

\section{Keywords:}

settlement;

climate;

geography; human growth;

West Africa

\begin{abstract}
Résumé - D'un peu plus de 30 millions d'habitants en 1900, la population ouest-africaine est passée à 306 millions d'habitants en 2010. D'une Afrique de l'Ouest faiblement peuplée et caractérisée par des paysages souvent indemnes d'action humaine, on est passé à une région densément peuplée, avec des paysages profondément marqués par l'anthropisation. Les trois faits significatifs de cette évolution ont été une forte densification de l'espace rural, une urbanisation rapide et une dégradation importante de l'environnement. La population de la région devrait continuer d'augmenter et se situer entre 550 et 700 millions en 2050. Il est donc intéressant de s'interroger sur l'évolution du peuplement, des paysages, et des relations déjà tendues entre populations et environnement dans cette région où les populations sont enclavées, et qui sera soumise à une variabilité climatique croissante.
\end{abstract}

\begin{abstract}
A century of settlement in West Africa. From some 30 million inhabitants in 1900 West African population has risen to 306 million in 2010. Within one century this under-populated region which used to display landscapes often unscarred by human action has turned into a highly anthropized, densely populated region. The three major factors of this evolution have been high rural density (from a maximum of 25 inhabitants $/ \mathrm{km}^{2}$ in 1892 to over 100 inhabitants $/ \mathrm{km}^{2}$ nowadays), rapid urbanization (from $10 \%$ of urban people in 1950 to $50 \%$ predicted for 2020) and a dramatic degradation of vegetation in savannah, forest and mangrove areas. The population will continue to grow and is expected to reach between 550 to 700 million inhabitants in 2050, depending respectively on low or high hypotheses. It is worthwhile speculating about the evolution of land settlement and landscapes and the already strained relationships between humans, resources and the environment in population enclaves which in addition will be subjected to high climatic variability. To prevent a generalization of conflicts and their fatal consequences on sustainable development (education, accommodation, health), it is advisable to accurately appraise the challenges Western Africa will have to face 50 years from now and also to become aware that none of these challenges can be met without taking into consideration the interrelations existing between them.
\end{abstract}

\section{Introduction}

La population de l'Afrique de $l^{\prime}$ Ouest ${ }^{1}$ est passée d'un peu plus de 30 millions d'habitants en 1900 à 306 millions

Auteur correspondant : F. Courtin, fabrice.courtin@ird.fr J.-P. Guengant a été le représentant de l'IRD au Burkina Faso d'octobre 2003 à septembre 2009 et en Côte d'Ivoire de janvier 2007 à septembre 2009.

1 Mauritanie, Sénégal, Gambie, Guinée-Bissau, Guinée, Liberia, Sierra Leone, Mali, Niger, Burkina Faso, Côte d'Ivoire, Ghana, Togo, Bénin, Nigeria. d'habitants en 2010 (United Nations, 2010). Cet accroissement démographique exceptionnel par son ampleur et sa rapidité s'est réparti sur une superficie de 6140000 km² de manière distincte, en fonction des disparités d'accroissement naturel et des principaux flux migratoires. Ainsi, les régions possèdent des densités de population variables selon leur histoire démographique, économique, épidémiologique, climatique et politique ${ }^{2}$. En

\footnotetext{
${ }^{2}$ Cette étude a été construite à partir d'une synthèse bibliographique et d'une restitution des observations faites lors des
} 
effet, que ce soit les migrations sahéliennes engendrées par l'économie de plantations de café et/ou de cacao en Côte d'Ivoire et au Ghana (Mandé, 1997), l'abandon des vallées soudaniennes du fait du couple épidémiologique trypanosomiase-onchocercose (Hervouët, 1990), la fuite vers le Sud des populations du Sahel après les sécheresses des années 1970 (Gallais, 1977) ou le retour forcé de milliers de rapatriés au Burkina Faso (Courtin et al., 2011), ces événements ont contribué à modeler le peuplement actuel de l'Afrique de l'Ouest. De nos jours, la multiplication des accidents climatiques (UNOCHA, 2009) et des conflits (UNOCHA, 2011) tend à rendre cette mobilité de plus en plus involontaire.

La croissance démographique et les courants migratoires qu'elle a en partie impulsés ont provoqué une densification de l'espace rural (OCDE, 1998), une expansion exceptionnelle des villes (AFD et SEDET, 2008) et une dégradation marquée de l'environnement (FAO, 2009). Entre 1950 et 2010, la population rurale a été multipliée par trois et la population urbaine par vingt-deux (United Nations, 2010) ! De massivement rurale en 1950, avec seulement $10 \%$ de sa population vivant dans les villes, l'Afrique de 1'Ouest aura dès 2020 autant d'urbains que de ruraux, et elle compte déjà dix-sept villes millionnaires dont huit au Nigeria (ibid.). L'augmentation des densités rurales a profondément modifié le paysage végétal et animal, de la savane burkinabè à la mangrove guinéenne, en passant par la forêt ivoirienne. Les faits les plus remarquables sont la quasi-disparition de la forêt ombro-mésophile ainsi que de la faune sauvage en savane et en forêt, qui ne subsiste plus que dans quelques rares zones bien protégées grâce à une gestion adaptée aux situations locales (Pendjari au Bénin, Niokolo-Koba au Sénégal, Taï en Côte d'Ivoire...). Les Ouest-Africains vivent aujourd'hui dans un contexte de saturation foncière, particulièrement en zone forestière.

La population va continuer d'augmenter fortement, puisqu'on estime qu'elle devrait se situer en 2050 entre 550 et 700 millions (ibid.). Ce phénomène démographique, au vu des constats effectués sur l'évolution du peuplement et des paysages au cours du $X X^{\mathrm{e}}$ siècle, risque de poser des problèmes, d'autant plus qu'il s'effectuera dans un contexte de forte variabilité climatique (AMMA, 2009).

Les évolutions annoncées sont susceptibles d'accentuer les tensions entre les différents utilisateurs des ressources foncières et hydriques (agriculteurs, éleveurs, pêcheurs, autochtones, allochtones, allogènes) ainsi qu'entre les bénéficiaires des ressources de l'État et de l'aide internationale (urbains et ruraux, communautés ethniques et religieuses diverses). L'objectif de cet article est de sensibiliser, par une mise en perspective historique des dynamiques de peuplement, les gouvernements et

recherches menées en Afrique de l'Ouest par les auteurs de cet article. les acteurs du développement aux défis que devra relever l'Afrique de l'Ouest.

\section{Causes de sous-peuplement et paysages coloniaux (1892-1960)}

Sur la carte établie par Binger en 1892, les densités de population en Afrique de l'Ouest s'échelonnent de 1 à 25 habitants par kilomètre carré ${ }^{3}$ (Fig. 1). Cette carte présente six principaux môles de peuplement précoloniaux ainsi que trois môles secondaires. Les principaux centres d'Afrique de l'Ouest sont alors de gros villages, tels que (selon l'orthographe utilisée par Binger) Saint-Louis, Konacry, Monrovia, Bammako, Sikasso, Waghadougou, Bobo-Dioulasou (ou Sia), Kong, Grand-Bassam, Coumassi, Porto-Novo, Kotonou. L'auteur écrit à propos de Waghadougou (Ouagadougou) : «J'estime que la population totale de tous ces groupes ne doit pas dépasser 5000 habitants » (Binger, 1892, p. 459).

En 1900, on estime la population ouest-africaine entre 30 et 35 millions d'habitants, soit une densité de population de 5 à $6 \mathrm{hab} / \mathrm{km}^{2}$. Ce sous-peuplement, c'est-àdire cette densité de population nettement inférieure aux potentialités du milieu, ne suggère pas seulement des techniques d'encadrement et de production inadaptées (Gourou, 1983). En effet, la traite négrière et les premières incursions européennes ont participé au déséquilibre des sociétés ouest-africaines et ont ainsi affecté leurs capacités à maîtriser leur environnement (productions agricoles, pathologies, faune sauvage...). Au-delà d'un taux élevé de mortalité infantile ou d'infertilité liée à certaines pathologies (gonococcie, trypanosomiase, schistosomiase, etc.), ce sous-peuplement était d'autant plus accentué que les conflits dépeuplaient certaines régions. Par exemple, avant la capture de Samory à Guélémou (région de Touba en Côte d'Ivoire), et à l'approche de son campement provisoire, le capitaine français Gouraud (1939, p. 199) écrit : «Dans tout le Soudan, la plupart d'entre nous ont vu depuis longtemps les traces du passage de l'Almamy, mais des traces vieilles, refroidies : la ruine, le vide, l'abandon. Ici c'est le royaume de la mort, de l'horreur ! Ce contexte d'insécurité générale fut réduit au fur et à mesure que les colonisateurs implantaient les postes militaires, comme par exemple dans le Sud-Ouest ivoirien (Thomann, 1999) ou dans le Fouta Djalon (Monénembo, 2008).

Parallèlement à ces troubles politiques, les épisodes de sécheresse provoquaient des disettes, comme celle qui dura de 1900 à 1903, appelée au Niger «Ize-Nere » (vente des enfants). Les témoignages recueillis en pays songhay (Tillabéry, Téra) évoquent des vagues successives de populations chassées déferlant sur les rives du fleuve Niger : « [...] les gens venaient de l'Anzuru ou du Zarmaganda

\footnotetext{
${ }^{3}$ Ces informations sont à relativiser compte tenu des faibles connaissances des populations et des territoires de l'époque.
} 


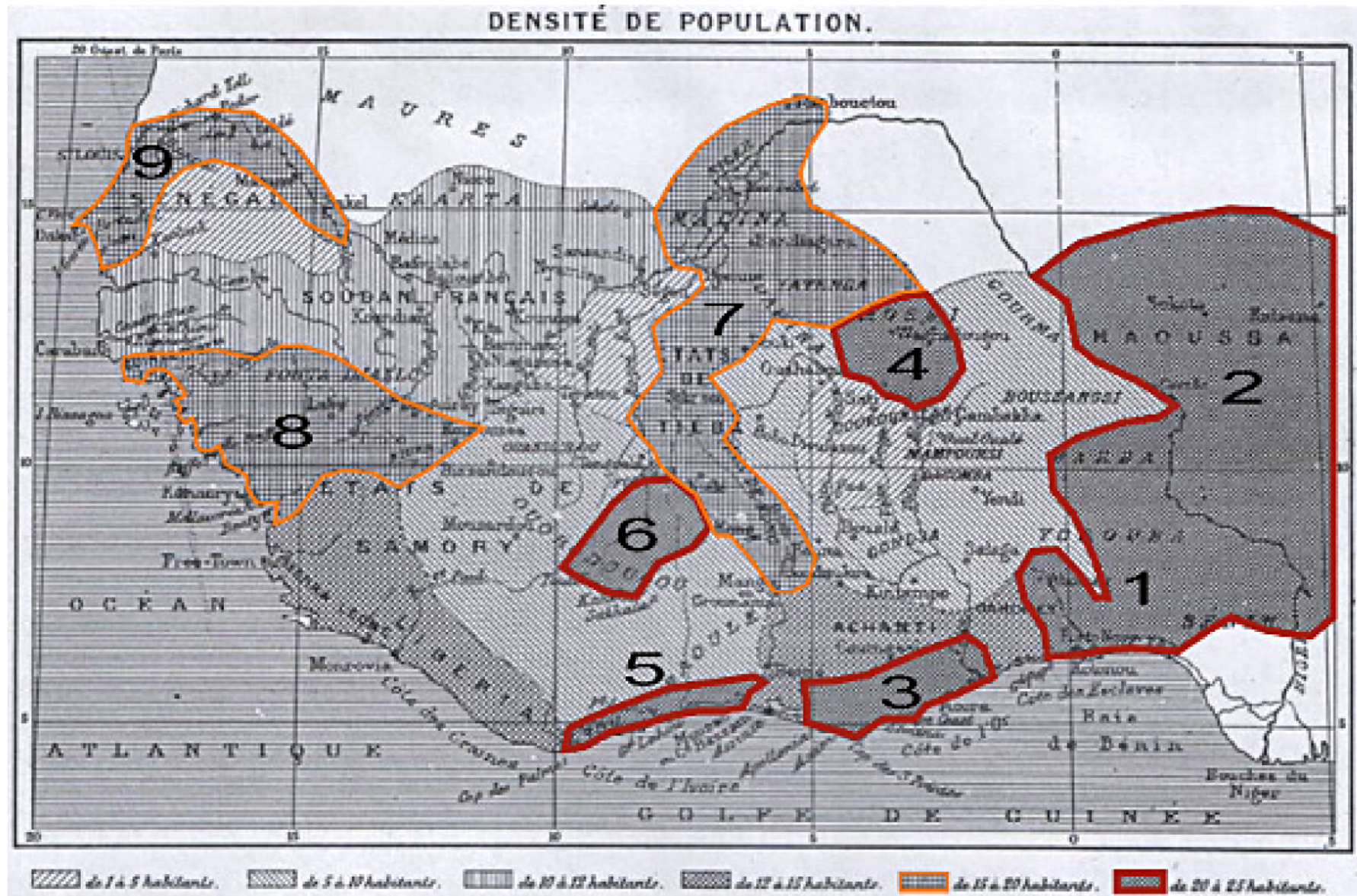

Fig. 1. Densité de population en Afrique de l'Ouest en 1892. (Source : Binger, 1892.)

Môles principaux : Yoruba (1), Haoussa (2), Fanti (3), Mossi (4), Krou-Alladian-Apollonien (5), Sénoufo (6) ; môles secondaires : Bambara (7), Peul (8), Wolof-Serer-Toucouleur (9).

et il en arrivait des centaines par jour avec leurs enfants qu'ils proposaient d'échanger contre un panier de grain » (in Gado, 1993, p. 74).

La période de «paix coloniale » qui suit voit l'installation des premières structures sanitaires, qui permettent aux autorités coloniales de prendre conscience du «fardeau pathologique» que supportent les populations africaines (Bado, 1996). Cet «intérêt » pour la santé des populations africaines va de pair avec les déplacements et les morts occasionnés par les travaux forcés pour l'aménagement (routes, ponts, bâtiments, wharfs, chemin de fer) et l'exploitation des territoires (bois précieux, plantations, mines), eux-mêmes favorables à la propagation des maladies (trypanosomiase, onchocercose...) [DomergueCloarec, 1986]. En 1929, le gouverneur de Côte d'Ivoire, Brunot, dénonce "un système illégal aux conséquences lamentables de travail forcé des indigènes pour les coupeurs de bois européens » et le fait terrible que « ces gens sont plus mal traités que s'ils étaient achetés. Achetés ils auraient une valeur, en effet, recrutés et remplacés d'office sans contrôle effectif possible par l'administration, ils n'en ont pas » (Brunot, 1929, p. 24).
En dépit des nombreux décès occasionnés par les guerres, famines, maladies et travaux forcés, la population d'Afrique de l'Ouest augmente, grâce à la réduction globale de la mortalité imputable à la mise en place de structures sanitaires et aux progrès de la médecine coloniale (Société de pathologie exotique, 2008). L'augmentation correspondante de la population est visible lorsqu' on compare la carte faite par Binger en 1892 et celle établie par l'Office de recherche scientifique coloniale (ORSC) en 1947 (Fig. 2). En effet, la carte de 1947 indique des densités de population qui s'échelonnent alors de moins de 1 à plus de $50 \mathrm{hab} / \mathrm{km}^{2}$, soit des densités maximales doubles de celles figurant sur la carte de 1892 et une évolution du peuplement avec des foyers qui se rejoignent et d'autres qui disparaissent. Ainsi, le môle Yoruba est désormais relié au foyer Fanti-Ashanti par l'intermédiaire du môle Fon. On note l'apparition de deux môles très localisés, en pays Kusasi et Somba. Dans le même temps, les pôles secondaires Toucouleur, Peul, Temne-MendeKissi, Baoulé et Sonraï se dessinent. Globalement, la population ouest-africaine est estimée en 1950 à 68 millions $\left(11 \mathrm{hab} / \mathrm{km}^{2}\right)$, soit le double de l'estimation du début du 


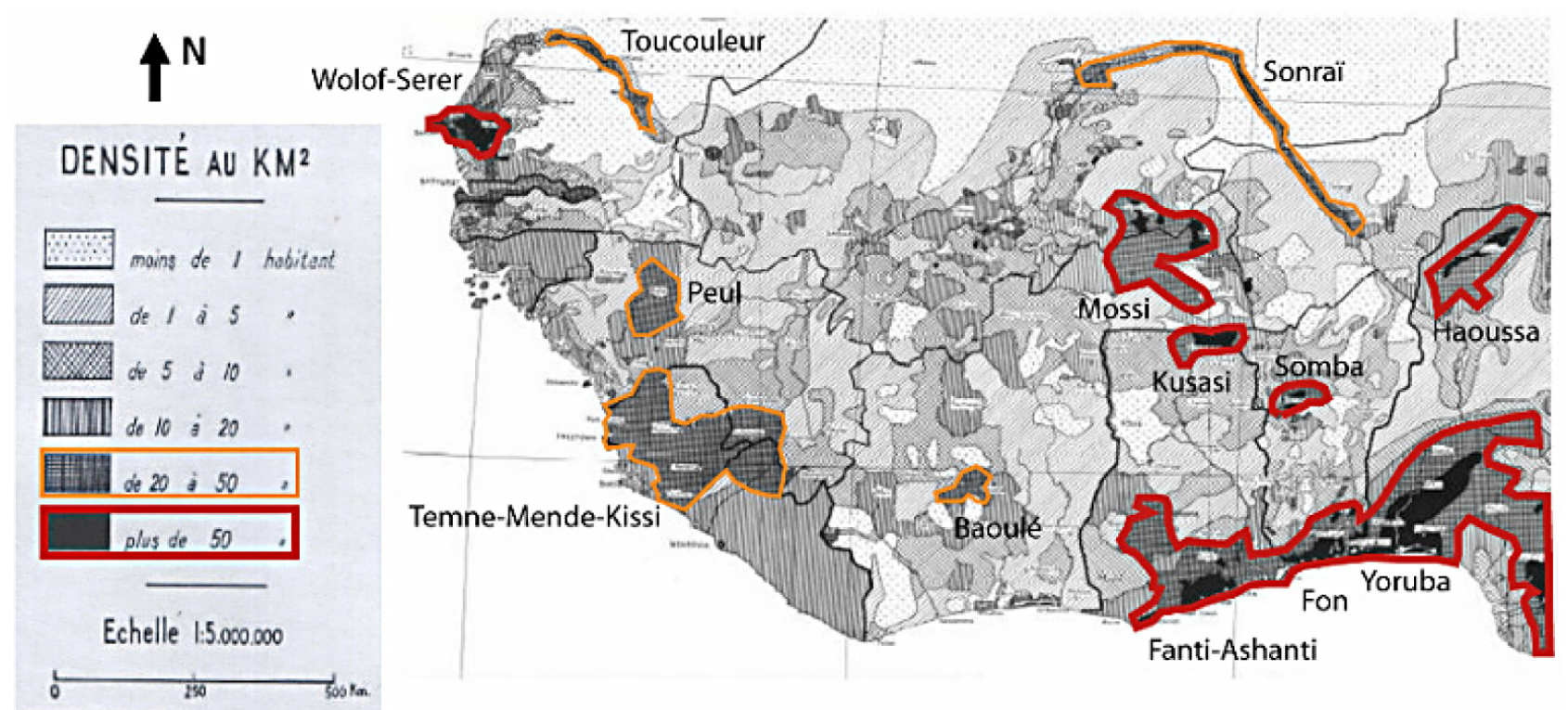

Fig. 2. Densité de population en Afrique de l'Ouest en 1947. (Source : ORSC, cartothèque de l'IRD.)

siècle (United Nations, 2010). Cette population est constituée de $90 \%$ de ruraux et de $10 \%$ d'urbains (ibid). La distribution de cette population a été finement étudiée par l'Institut français d'Afrique noire (IFAN), sous l'impulsion de Théodore Monod, à travers l'élaboration de cartes ethno-démographiques de l'Afrique-Occidentale qui confirment les observations de l'ORSC (Suremain, 2008).

\section{Accélération des dynamiques de peuplement et des paysages (1960-2009)}

Puis la croissance démographique s'accélère et, en 1960, la population d'Afrique de 1'Ouest est estimée à 84 millions d'habitants, soit une densité de population de $14 \mathrm{hab} / \mathrm{km}^{2}$ (United Nations, 2010). Un des facteurs majeurs du peuplement contemporain d'Afrique de l'Ouest a été la sécheresse des années 1970 (L'Hôte et Mahé, 1996). Elle a eu comme conséquence une dégradation poussée des paysages sahéliens, usés au possible par les paysans et les éleveurs du fait de systèmes agraires extensifs, avant que ceux-ci ne se décident à fuir vers le Sud (Gallais, 1977). L'alternative se trouvait alors vers d'autres régions rurales mieux drainées, par exemple vers les vallées de la bande soudanienne libérées de l'onchocercose grâce à l'Onchocerciasis Control Program (OCP) [Quesnel et al., 1999], ou vers les villes touchées par l'épidémie du VIH/SIDA (Vennetier, 1991). Cette dernière n'a réduit que marginalement la croissance démographique de l'Afrique de l'Ouest, qui a été la région d'Afrique subsaharienne la moins affectée, avec des prévalences situées entre 1 et $5 \%$ et un nombre de personnes vivant avec la maladie estimé à 4 millions en 2007
(ONUSIDA, 2008). Malgré ces contraintes, on compte, en 2010, 306 millions d'habitants (50 hab $\left./ \mathrm{km}^{2}\right)$, parmi lesquels 156 millions de ruraux (55\%) et 114 millions d'urbains (45\%) [United Nations, 2010]. L'urbanisation est le fait marquant de ce peuplement avec l'existence, en 2010, de dix-sept villes millionnaires (dont huit situées au Nigeria), tandis que Ouagadougou est devenue une capitale peuplée de près de 2 millions d'habitants. Les quatre villes les plus importantes sont Lagos (10 millions), Abidjan (4 millions), Kano (3 millions) et Dakar (2,5 millions) [ibid.].

Sur la figure 3, sont représentées les villes millionnaires ainsi que les principaux foyers de peuplements d'Afrique de 1'Ouest, à travers 160000 entités géoréférencées représentant les sites d'implantations humaines (NGA, 2008). Les foyers de peuplement contemporains apparaissent et confirment la constance dans l'évolution du peuplement précolonial. Pour chacun des pays côtiers d'Afrique de l'Ouest, la ville la plus importante est située sur la façade maritime, principalement du fait d'une pluviométrie propice aux cultures industrielles, de la présence de ports favorables au transport de marchandises et de la proximité de ressources pétrolières offshore situées dans le golfe de Guinée, notamment au Nigeria. Les zones de très faible peuplement se superposent généralement aux espaces protégés encore bien conservés.

La croissance de la population (Fig. 4), accompagnée d'une augmentation importante du cheptel bovin - véritables «billets de banque sur pied » (Bernus, 1995) -, a conduit à une dégradation importante de la végétation arborée en Afrique de l'Ouest. C'est la région d'Afrique où la vitesse de dégradation de cette végétation est la plus rapide $(-1,17 \%$ par an) [FAO, 2009]. En forêt, on a assisté dans un premier temps à une déforestation sélective pour 


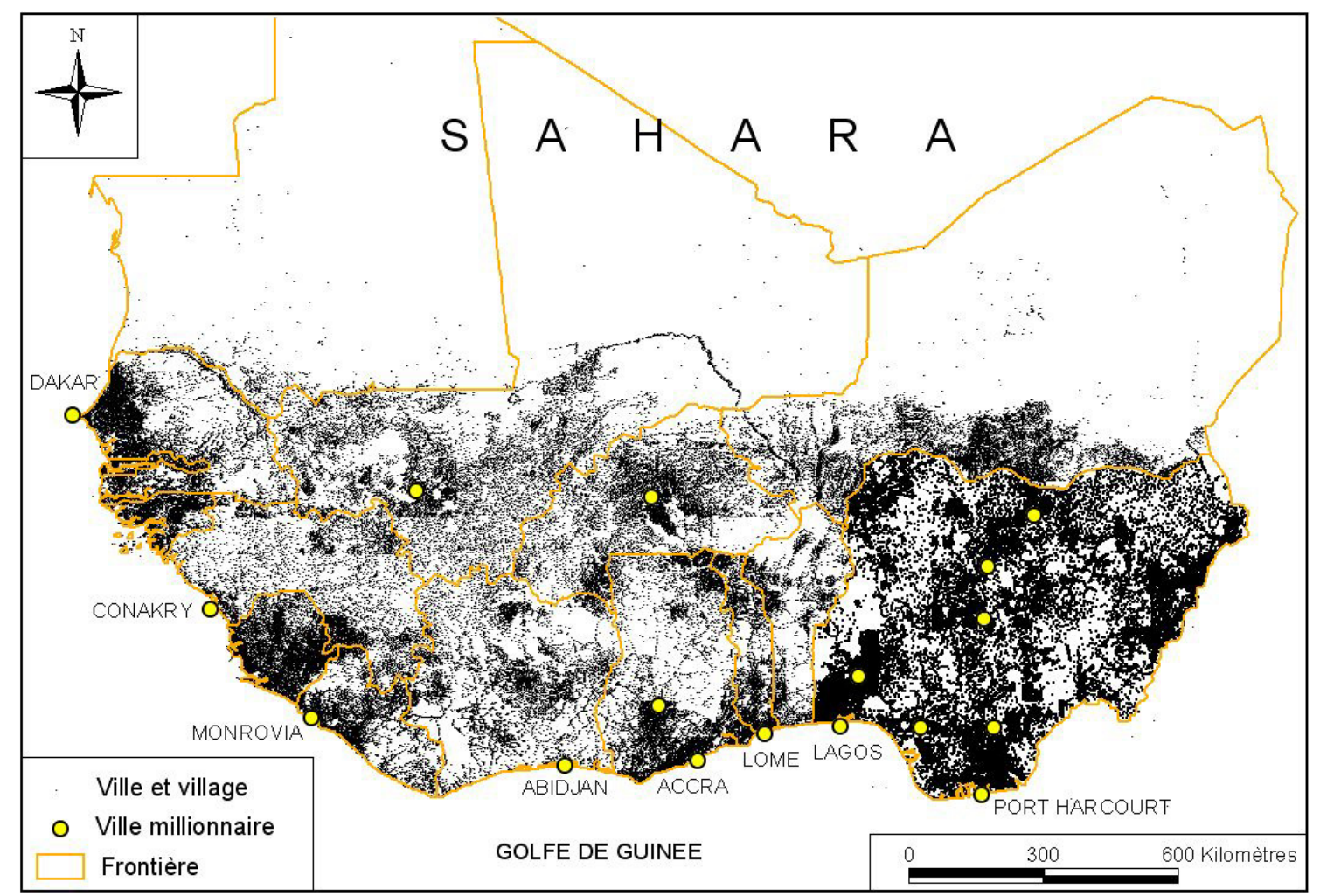

Fig. 3. Foyers contemporains de peuplement en Afrique de l'Ouest. (Sources : NGA, 2008 ; United Nations, 2010.)

l'exploitation des bois précieux (acajou, iroko...). Cette dernière a été suivie par une coupe générale pour la mise en place de cultures industrielles (ananas, hévéa...) ou de plantations familiales (café, cacao...), généralement associées à des cultures vivrières (riz, taro...). Les migrations en provenance de la bande sahélienne ont nettement participé à l'augmentation rapide des densités de population et, avec elle, à la disparition de la forêt (Boni, 1978). Aujourd'hui, la plupart des forêts classées et des parcs nationaux situés en zone de forêt sont devenus ce qu'on appelle communément en Côte d'Ivoire du " café-cacao classé », à l'exemple du Parc national de la Marahoué. La compétition pour l'accès à la terre pose de plus en plus la question de son appartenance et génère des tensions entre autochtones, allochtones et allogènes, à l'instar de ce qui est observé dans le Centre-Ouest ivoirien entre Gouro, Baoulé et Mossi (Chauveau et Bobo, 2003).

En savane, l'augmentation de la population a également contribué à l'extension des surfaces cultivées : développement des cultures de rente (coton, arachide...) et vivrières (mil, sorgho...). La première conséquence de cette anthropisation brutale a été la disparition de la faune sauvage, excepté dans quelques espaces bien protégés tels que ceux du complexe W-Arly-Pendjari (Niger-Burkina Faso-Bénin). De nombreuses forêts classées sont devenues des champs et il est à craindre que les derniers espaces protégés ne constituent les fronts pionniers de demain. Cette pression anthropique accroit le nombre de conflits entre agriculteurs et éleveurs, comme en 2008 entre des éleveurs peuls et des agriculteurs lobi dans le Sud-Ouest du Burkina Faso (région de Gaoua).

En mangrove, on retrouve ces processus de dégradation liés à l'augmentation des densités humaines (Cormier-Salem, 1999). La surface occupée par les palétuviers dans la région est ainsi passée de 2051900 hectares en 1980 à 1787200 en 2005 (FAO, 2007). Un des exemples les plus illustratifs est le cas de la Guinée maritime, où, de 1980 à 2005, la superficie occupée par les palétuviers a diminué de $11 \%$ (ibid.). Les activités de charbonnage ont provoqué une importante dégradation des sols situés autour des villages localisés sur l'interface savanemangrove et réorienté les populations vers la mangrove. Parallèlement, la présence croissante d'éleveurs peuls et de leurs troupeaux dans la plupart des villages littoraux provoque des conflits entre agriculteurs (Soussou, Baga) et éleveurs (Peuls), comme celui survenu dans la souspréfecture de Forécariah en 2007.

Les exemples ci-dessus illustrent bien comment cet accroissement démographique a modifié le peuplement, le paysage et les relations entre les différentes communautés utilisatrices des ressources. Pourtant, la population de la région va continuer à croître fortement dans les décennies à venir, même si, comme le supposent les Nations unies, le nombre moyen d'enfants par femme passe de 5,3 aujourd'hui à 2 ou 3 en 2050 - hypothèses qui paraissent très optimistes, voire peu réalistes (Guengant et 
Afrique de l'Ouest

pyramides des âges en 1960, 1990, 2010

1960: 75 millions ; 1990: 183 millions ; 2010 : 306 millions

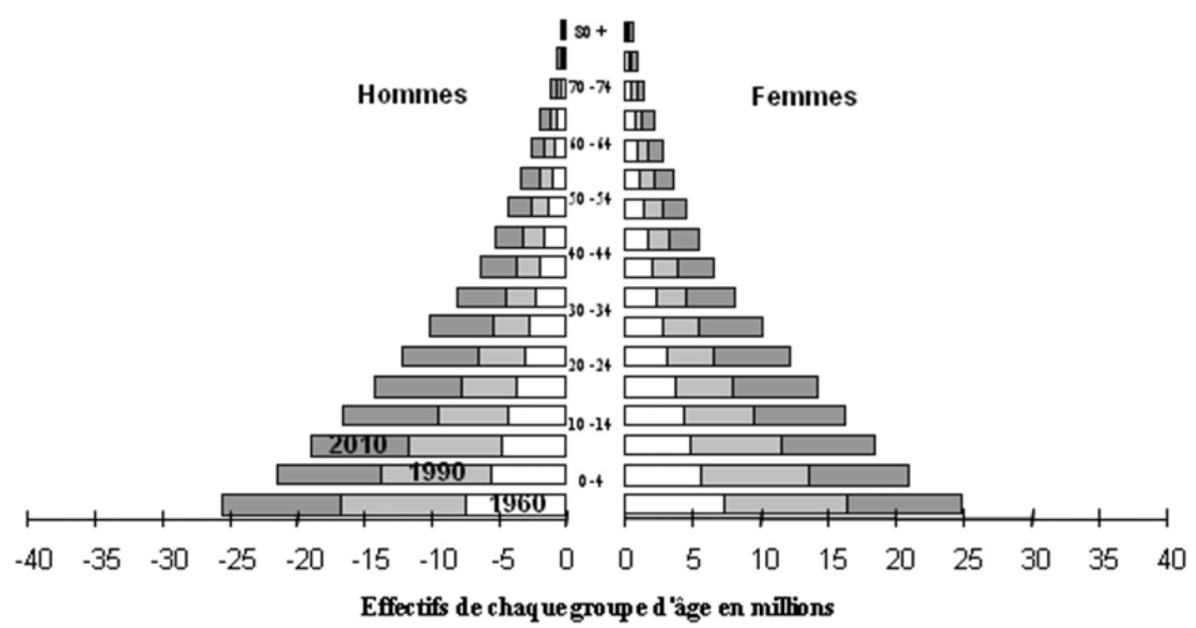

Prévisions 2050

hypothèse basse: 551 millions

hypothèse haute : 705 millions

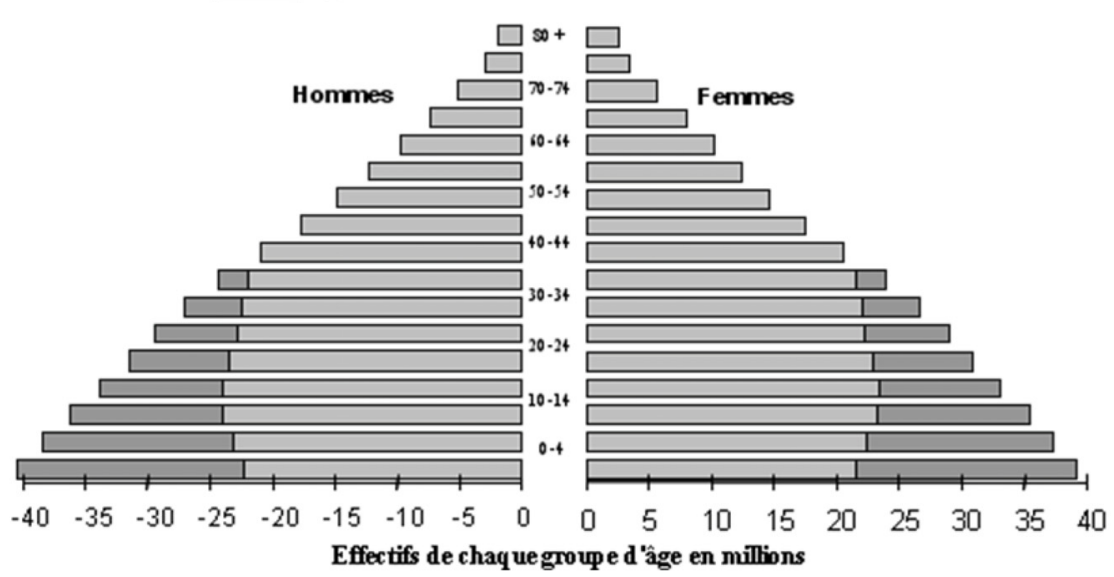

Fig. 4. Croissance démographique passée et future en Afrique de l'Ouest. (Source : United Nations, 2010.)

May, 2009). En effet, d'une part, la baisse de la fécondité est lente et encore modeste dans la quasi-totalité des pays de la région ( $c f$. Tab.) ; d'autre part, l'extrême jeunesse de la population (deux Ouest-Africains sur trois ont moins de 25 ans) signifie que la population totale et la population active vont continuer à croître fortement, même si la fécondité baisse très rapidement - ce qui, à nouveau, est loin d'être acquis (Fig. 4). L'indépendance fragile des femmes, la lente évolution des mentalités (culturelles, religieuses) sur le nombre d'enfants à avoir et les difficultés financières des femmes pour recourir ou accéder à la contraception, notamment en milieu rural, sont des facteurs qui continueront à jouer en faveur $\mathrm{d}^{\prime} \mathrm{un}$ fort taux de fécondité. L'évolution des taux de fécondité entre 19751980 et 2005-2010 des cinq pays à « transition très lente, voire non amorcée » (Tab.) illustre la persistance de ces obstacles. La plupart de ces pays ont des taux d'urbanisation faibles et ont connu des troubles intérieurs graves qui ont désorganisé leurs services publics et de santé.

Il faut souligner ici qu'une croissance démographique aussi forte est sans précédent dans l'histoire des populations humaines. Elle est la conséquence d'une baisse très rapide de la mortalité et du maintien sur une période exceptionnellement longue d'une forte fécondité. Avec une espérance de vie à la naissance estimée à 53 ans en 2010 (contre 38 ans en 1960) et une fécondité supérieure à cinq enfants par femme en 2010, la première phase de la transition démographique - la baisse de la mortalité est bien amorcée; alors que la seconde phase - la baisse de la fécondité - l'est à peine, d'où l'accroissement démographique exceptionnel constaté. Du fait de l'importance de la population jeune (Fig. 4), même si le nombre 
Tableau. Types de transition selon l'importance de la baisse de la fécondité en Afrique de l'Ouest entre 1975-1980 et 2005-2010. (Source : United Nations, 2010.)

\begin{tabular}{|c|c|c|c|c|c|}
\hline \multirow{2}{*}{ Pays } & \multicolumn{4}{|c|}{ Indice synthétique de fécondité (ISF) } & \multirow{2}{*}{$\begin{array}{l}\text { Différence } \\
2005-2010 \\
1975-1980\end{array}$} \\
\hline & $1975-1980$ & $1985-1990$ & $1995-2000$ & $2005-2010$ & \\
\hline \multicolumn{6}{|c|}{ Transition récente relativement bien amorcée } \\
\hline Cap-Vert & 6,8 & 5,9 & 4,1 & 3,4 & $-3,4$ \\
\hline Côte-d'Ivoire & 7,4 & 6,9 & 5,6 & 4,5 & $-3,0$ \\
\hline Ghana & 6,7 & 6,1 & 4,8 & 3,8 & $-2,8$ \\
\hline \multicolumn{6}{|c|}{ Transition plus lente et irrégulière } \\
\hline Sénégal & 7,0 & 6,8 & 5,7 & 4,7 & $-2,3$ \\
\hline Togo & 7,1 & 6,6 & 5,8 & 4,8 & $-2,3$ \\
\hline Mauritanie & 6,4 & 6,0 & 5,3 & 4,4 & $-2,0$ \\
\hline Gambie & 6,6 & 6,2 & 5,5 & 4,7 & $-1,9$ \\
\hline Burkina Faso & 7,8 & 7,4 & 6,8 & 6,0 & $-1,8$ \\
\hline Bénin & 7,1 & 6,9 & 6,3 & 5,4 & $-1,7$ \\
\hline Nigeria & 6,9 & 6,8 & 6,3 & 5,3 & $-1,6$ \\
\hline Guinée & 7,0 & 6,8 & 6,2 & 5,4 & $-1,6$ \\
\hline \multicolumn{6}{|c|}{ Transition très lente, voire non amorcée } \\
\hline Mali & 7,6 & 7,5 & 7,2 & 6,5 & $-1,0$ \\
\hline Niger & 8,1 & 8,0 & 7,7 & 7,2 & $-0,9$ \\
\hline Liberia & 6,9 & 6,9 & 6,8 & 6,8 & $-0,1$ \\
\hline Sierra Leone & 6,5 & 6,5 & 6,5 & 6,5 & 0,0 \\
\hline Guinée Bissau & 7,1 & 7,1 & 7,1 & 7,1 & 0,0 \\
\hline
\end{tabular}

d'enfants par femme s'établissait à deux rapidement, la population de l'Afrique de l'Ouest atteindrait tout de même 550 millions en 2050 ; et si le nombre d'enfants par femme s'établissait à trois, elle atteindrait 705 millions, contre 306 millions aujourd'hui (United Nations, 2010).

\section{Quels peuplements, quels paysages demain?}

D'ici quarante ans, donc, la population de l'Afrique de l'Ouest sera en gros deux à trois fois plus nombreuse qu'aujourd'hui, et elle sera au deux tiers urbaine, puisqu'on estime qu'en 2050, $68 \%$ de sa population vivra dans des villes (ibid.), dont plusieurs dizaines de villes millionnaires. Dans ce contexte, il est important de s'interroger sur l'évolution du peuplement, des paysages et des relations déjà tendues entre populations et environnement dans la région. Comment nourrir, soigner, loger et éduquer des populations beaucoup plus nombreuses qu'aujourd'hui ? Comment obtenir une stabilité politique à long terme dans une région où cette dernière a déjà été soumise à rude épreuve (Liberia, Sierra Leone, Côte d'Ivoire.) ? Qu'adviendra-t-il des espaces protégés?

Les constats effectués sur la dynamique du peuplement colonial, associés à la tendance postcoloniale présentée dans l'étude West Africa Long-Term Perspective Study (WALTPS) [OCDE, 1998], laissent à penser que la dynamique du peuplement ouest-africain continuera d'évoluer dans la même direction. Quelques variations locales pourraient apparaître $d u$ fait d'accidents climatiques qui seront plus nombreux et surtout plus problématiques, car ils se dérouleront dans des régions plus densément peuplées (Orgeval, 2008). Si les démographes veulent évaluer le poids de la population future d'Afrique de 1'Ouest et les géographes, sa distribution et le paysage dans lequel elle s'inscrira, ils se doivent de prendre en compte l'évolution du climat en général, et de la pluviométrie en particulier. Des programmes comme l'Analyse multidisciplinaire de la mousson africaine (AMMA, 2009), devraient permettre de répondre précisément à ces questions essentielles. La campagne de sensibilisation lancée par la FAO pour lutter contre la faim dans le monde concerne évidemment l'Afrique de l'Ouest. Le cas du Niger est à ce propos préoccupant, puisque sa population doit être multipliée au minimum par cinq au cours des cinquante prochaines années, alors que plus de la moitié des terres cultivables sont utilisées (Guengant et Banoin, 2003). Il faut donc que cette augmentation de la population s'accompagne (et c'est le cas) de la mise en place de nouveaux modes de production, afin d'améliorer la productivité des exploitations agricoles, à l'image des tracteurs qui labourent sous les parcs à Faidherbia albida dans le Sud-Ouest du Burkina Faso. Les modes de production animale évolueront probablement vers un élevage intensif, car le mode pastoral sera de plus 
en plus socialement (ethniquement) confronté au monde agricole dans les années à venir, du fait d'une rétraction de l'espace utile.

Ces nouveaux modes de production devront être viables, c'est-à-dire allier productivité et durabilité écologique, économique et sociale. Il s'agit donc d'inventer une "double révolution verte", issue de la recherche agricole, qui devra s'inspirer des connaissances locales (Griffon et Weber, 1996). Les capacités d'innovation et d'adaptation des acteurs qui font vivre les marchés ouest-africains (où est écoulé le vivrier marchand) joueront aussi un rôle primordial dans l'alimentation future des habitants (Chaléard, 1996). Ces capacités, en grande partie liées à la jeunesse de la population, qui se retrouve en ville dans le secteur informel (Touré, 1985) ou dans la mise en place d'incubateurs d'entreprises (IRD, 2009), constituent l'espoir de la région.

\section{Conclusion}

D'une densité de 5 hab/ $\mathrm{km}^{2}$ en 1900, l'Afrique de l'Ouest a atteint le chiffre de $50 \mathrm{hab} / \mathrm{km}^{2}$ en 2010 et passera à une densité comprise entre 90 et $115 \mathrm{hab} / \mathrm{km}^{2}$ en 2050 (United Nations, 2010). Ainsi, sa population aura été multipliée par au moins dix-huit en 150 ans. Par contraste, l'Europe et la Chine ont vu leurs populations multipliées seulement par cinq en quatre siècles (entre 1500 et 1900), ce qui, dans un contexte d'espace disponible important (Amériques, Afrique...), leur a laissé le temps et l'espace pour s'adapter. Par ailleurs, la réponse au défi alimentaire posé par la forte croissance démographique qu'ont connue, dans la seconde moitié du $X X^{\text {e }}$ siècle, les pays en développement d'Asie et d'Amérique a été la révolution verte, caractérisée par une forte mécanisation, 1'utilisation d'engrais chimiques et le recours à de nouvelles variétés. Mais celle-ci apparaît aujourd'hui non durable car dommageable pour l'environnement.

En Afrique de l'Ouest, par contre, l'augmentation de la production agricole s'est faite principalement par l'extension des surfaces cultivées, sans changement majeur des itinéraires techniques traditionnels à productivité faible, ce qui a été possible grâce aux faibles densités de population initiales. Ce type d'adaptation a atteint ses limites, car la terre autrefois abondante est devenue rare et source de conflits (Guengant, 2007).

Dans un contexte de crise alimentaire qui concerne à présent autant les populations urbaines que rurales, les systèmes agraires traditionnels ne seront pas en mesure de produire suffisamment pour une population deux ou trois fois plus nombreuse et de plus en plus urbaine. Il est donc urgent pour l'agriculture ouest-africaine d'inventer et de mettre rapidement en œuvre une révolution «doublement verte », qui alliera durabilité et augmentation majeure des rendements. Cela ne sera pas facile, surtout dans un contexte de variabilité climatique croissante, et ce d'autant plus qu'il n'est pas certain que l'appui que la communauté internationale est prête à apporter aux pays les plus vulnérables sera à la hauteur des besoins en la matière (comme on a pu le constater fin 2009, à l'occasion de la conférence de Copenhague sur le climat).

Existe-t-il une autre alternative? Certains auteurs (Collier, 2008) pensent qu'avec la hausse des coûts de la main-d'œuvre en Asie (et le vieillissement de la population chinoise), l'Afrique pourrait devenir le prochain " atelier du monde » compte tenu de la croissance rapide de sa propre main-d'œuvre. Ces activités manufacturières seraient plutôt localisées dans les zones maritimes qui pourraient recevoir les quantités de nourritures nécessaires. En tout état de cause, même si une partie de la nourriture des Africains continuera à être importée de l'extérieur du continent, l'Afrique ne peut pas faire l'économie d'une révolution agricole que certaines firmes multinationales étrangères n'hésitent d'ailleurs pas à exporter sur le continent, sans forcément en faire bénéficier les Africains.

Du point de vue de la distribution spatiale, les noyaux de forte ou de très forte densité de population restent aujourd'hui ceux où la croissance démographique est la plus importante. La migration vers les terres neuves a été un moyen de réduire la pression démographique dans les zones de forte densité, mais cette solution est de moins en moins possible du fait de la saturation foncière généralisée des espaces ruraux. Il en résulte une multiplication des conflits, qui tendent même à devenir la règle dans le haut de l'échelle des densités rurales (Barrau et al., 1983). Les migrations vers la ville ont atténué la densification des espaces ruraux, mais elles posent des problèmes de plus en plus difficiles de gestion et d'aménagement des espaces urbains (logements, transports, eau potable, santé...) [Fournet et al., 2008].

Pour empêcher la généralisation des tensions et conflits, il convient de prendre la juste mesure des défis auxquels l'Afrique de l'Ouest est et continuera d'être confrontée dans les cinquante prochaines années, et aussi de prendre conscience qu'aucun de ces défis ne pourra être relevé isolément, c'est-à-dire sans prise en considération des interrelations existant entre eux. Il s'agit en particulier, dans l'ordre alphabétique, des défis alimentaire, climatique, démographique et sanitaire. La double révolution verte à mettre en œuvre ne sera durable que si elle anticipe les conséquences négatives des changements climatiques sur la pluviométrie et la fertilité des sols, et si, parallèlement, on observe une décélération de la forte croissance démographique actuelle. L'adaptation aux changements climatiques et la résilience des populations face aux conséquences d'événements naturels extrêmes (sécheresses, inondations) supposent des populations éduquées, informées, en bonne santé et bénéficiant d'une nourriture suffisante et équilibrée, ce qui 
ne pourra se faire tant que l'occupation de l'espace dans les zones urbaines et rurales ne sera pas planifiée (Guengant, 2011).

L'adoption et la mise en œuvre rapide de politiques et de programmes dans les domaines alimentaire, climatique et démographique, coordonnés entre eux et avec ceux d'autres domaines, détermineront, in fine, le degré de stabilité politique et sociale des pays de la région, et le succès ou l'échec des politiques actuelles de lutte contre la pauvreté. La maladie, l'analphabétisme, la faim, la guerre et la pauvreté ne sont pas une spécificité africaine, et encore moins une fatalité (Severino et Ray, 2010).

\section{Remerciements}

Les auteurs remercient Jean Boutrais et Catherine Aubertin, ainsi que Philippe Solano et Vincent Jamonneau pour leurs conseils et la relecture du manuscrit.

\section{Références}

AFD, SEDET, 2008. Dynamiques de l'urbanisation, 1950-2020: approche géo-statistique, Afrique de l'Ouest. Rapport Africapolis, Paris.

AMMA, 2009. Third African Monsoon Multidisciplinary Analyses. Rapport AMMA, Ouagadougou.

Bado, J.-P., 1996. Médecine coloniale et grandes endémies en Afrique (1900-1960) : lèpre, trypanosomiase humaine et onchocercose, Paris, Karthala.

Barrau, J., Gourou, P., Sautter, G., 1983. Populations, civilisations et sociétés humaines : les densités de population, in Fournier, F., Sasson, A. (Eds), Écosystèmes forestiers tropicaux d'Afrique, Paris, ORSTOM/UNESCO, 351-372.

Bernus, E., 1995. Pasteurs face à la sécheresse : rebondir ou disparaître?, Revue de géographie de Lyon, 70, 3-4.

Binger, L.G., 1892. Du Niger au golfe de Guinée, par le pays de Kong et le Mossi (1887-1889), Paris, Hachette.

Boni, D., 1978. Aspects géographiques du binôme café-cacao dans l'économie ivoirienne, Abidjan, Nouvelles éditions africaines.

Brunot, R., 1929. Lettre de l'administrateur en chef des colonies Richard Brunot à monsieur le ministre des colonies. Rapport du gouverneur par intérim de Côte d'Ivoire, Centre d'archive Outre-mer, Aix-en-Provence.

Chaléard, J.-L., 1996. Marchés et vivrier marchand en Afrique occidentale : le cas de la Côte d'Ivoire, Historiens et géographes, 379, 111-122.

Chauveau, J.-P., Bobo, K.S., 2003. La situation de guerre dans l'arène villageoise, un exemple dans le Centre-Ouest ivoirien, Politique africaine, 89, 12-32.

Collier, P, 2008. The Bottom Billion: Why the Poorest Countries Are Failing and What Can Be Done About It, Oxford and New York, Oxford University Press.

Cormier-Salem, M.-C., 1999. Appropriation des ressources, enjeu foncier et espace halieutique sur le littoral ouest-africain, in Chauveau, J.-P., Jul-Larsen, E., Chaboud, C. (Eds), Les Pêches piroguières en Afrique de l'Ouest: pouvoirs, mobilités, marchés, Paris, Karthala, 205-229.
Courtin, F., Fournet, F., Solano, P., 2011. La crise ivoirienne et les migrants burkinabés : l'effet boomerang d'une migration internationale, Afrique contemporaine, 236, 11-27.

Domergue-Cloarec, D., 1986. La Santé en Côte d'Ivoire (19051958). Thèse de doctorat d'État en histoire, Université Toulouse-Le Mirail, Toulouse.

FAO, 2007. The World's Mangroves, 1980-2005, Africa. Rapport FAO, Rome.

FAO, 2009. State of the World's Forests, Africa. Rapport FAO, Rome.

Fournet, F., Meunier-Nikiema, A., Salem, G., 2008. Ouagadougou (1850-2004) : une urbanisation différenciée, Marseille, IRD Éditions.

Gado, B.A., 1993. Une histoire des famines au Sahel : étude des grandes crises alimentaires (XIX ${ }^{e}-X X^{e}$ siècles), Paris, L'Harmattan.

Gallais, J., 1977. Stratégies pastorales et agricoles des Sahéliens durant la sécheresse 1969-1974. Travaux et documents de géographie tropicale, CEGET-CNRS, Bordeaux.

Gouraud, H., 1939. Au Soudan, Paris, Pierre Tisné.

Gourou, P., 1983. Terres de bonnes espérances : le monde tropical, Paris, Plon.

Griffon, M., Weber, J., 1996. La révolution doublement verte : économie et institutions, Cahiers Agricultures, 5, 4, 239-242.

Guengant, J.-P., 2007. La démographie africaine entre convergences et divergences, in Ferry, B. (Ed.), L'Afrique face à ses défis démographiques : un avenir incertain, Paris, AFD/Karthala / Nogent-sur-Marne, CEPED-25-121.

Guengant, J.-P., 2011. Comment bénéficier du dividende démographique? La démographie au centre des trajectoires de développement dans les pays de l'UEMOA ainsi qu'en Guinée, au Ghana, en Mauritanie et au Nigeria : synthèse régionale, Paris, Agence française de développement. Version non définitive publiée à l'occasion de la conférence Population, développement et planification familiale en Afrique de l'Ouest francophone : l'urgence d'agir, Ouagadougou, 8-11 février 2011 (http:// www.afd.fr/jahia/Jahia/home/publications/Publicationsthematiques-geographiques/conference-ouagadougou).

Guengant, J.-P., Banoin, M., 2003. Dynamique des populations, disponibilités en terres et adaptation des régimes fonciers : le cas $d u$ Niger, Rome, FAO/CICRED.

Guengant, J.-P., May, J.F., 2009. Proximate determinants of fertility in sub-Saharan Africa and their possible use in fertility projection, United Nations Expert Group Meeting on Recent and Future Trends in Fertility, 24 December 2009, Population Division, United Nations, New York (http://www.un.org/esa/population/meetings/ EGM-Fertility2009/egm-fertility2009.html).

Hervouët, J.-P., 1990. Le mythe des vallées dépeuplées par l'onchocercose, Cahiers GEOS, 18, 1-35.

IRD (Institut de recherche pour le développement), 2009. L'Afrique de l'Ouest lance son premier incubateur d'entreprise : http://www.ird.fr/toute-1-actualite/actualites/ communiques-et-dossiers-de-presse/l-afrique-de-l-ouestlance-son-premier-incubateur-d-entreprises.

L'Hôte, Y., Mahé, G., 1996. Afrique de l'Ouest et centrale, précipitations moyennes annuelles (période 1951-1989), départements eaux continentales, laboratoire de cartographie appliquée, ORSTOM : http://www.cartographie.ird.fr/images/ pluvio_afrique/pluvio3.gif. 
Mandé, I., 1997. Les Migrations du travail en Haute-Volta (actuel Burkina Faso) : mise en perspective historique (1919-1960). Thèse de doctorat en histoire, Université Denis Diderot, Paris.

Monénembo, T., 2008. Le Roi de Kahel, Paris, Seuil.

NGA (National Geospatial-Intelligence Agency), 2008. Geonet, Afrique de l'Ouest: http://earth-info.nga.mil/gns/html/ namefiles.htm.

OCDE, 1998. Pour préparer l'avenir de l'Afrique de l'Ouest, une vision à l'horizon 2020, Paris, OCDE.

ONUSIDA, 2008. Rapport sur l'épidémie mondiale de sida, ONUSIDA, Genève (http://www.unaids.org/fr/resources/ unaidspublications/2008/).

Orgeval, T. d', 2008. Impact du changement climatique sur la saison des pluies en Afrique de l'Ouest : que nous disent les modèles de climat actuels ?, Sécheresse, 19, 79-85.

Quesnel, A., Vignikin, K., Zanou, B., N'Guessan, K., Vilquin, E., 1999. Dynamique de peuplement des zones rurales libérées de l'onchocercose en Afrique de l'Ouest, Paris, CICRED.

Severino, J.-M., Ray, O., 2010. Le Temps de l'Afrique, Paris, Odile Jacob.

Société de pathologie exotique, 2008. Bulletins, sommaires et résumés : http://www.pathexo.fr/bull_sommaire.php?L=1.
Suremain, M.-A. de, 2008. Les cartes ethno-démographiques de l'Afrique occidentale : enjeux d'une construction 1952-1963, Mappemonde, 92, 4 (http://mappemonde.mgm.fr/num20/ articles/art08404.html).

Thomann, G., 1999. Carnets de route en Côte d'Ivoire (1893-1902), Saint-Maur-des-Fossés, SEPIA.

Touré, A., 1985. Les Petits Métiers d'Abidjan : l'imagination au secours de la conjoncture, Paris, Karthala.

United Nations, 2010. World Population Prospects: The 2010 Revision, Population Database, United Nations Population Division, New York (http://esa.un.org/unpd/wpp/index. htm).

UNOCHA (United Nations Office for the Coordination of Humanitarian Affairs), 2009. Flood affected population, June to September 2009 (http://reliefweb.int/sites/reliefweb. int/files/resources/14D93E78C3482C2F8525764200666310map.pdf).

UNOCHA (United Nations Office for the Coordination of Humanitarian Affairs), 2011. West Africa 2011: Consolidated Appeals. Report, Unitied Nations, New York / Palais des Nations, Geneva (http://reliefweb.int/sites/reliefweb.int/ files/resources/CC24219E6B969A6C852577EB0057CB57Full_Report.pdf).

Vennetier, P., 1991. Les Villes d'Afrique tropicale, Paris, Masson.

Reçu le 15 janvier 2010. Accepté le $1^{\text {er }}$ décembre 2010. 